\title{
НЕКОТОРЫЕ ПРОБЛЕМЫ ФИЗИКО-ХИМИЧЕСКОГО АНАЛИЗА ДВОЙНЫХ ЖИДКИХ СИСТЕМ, ОДИН КОМПОНЕНТ КОТОРЫХ СКЛОНЕН К САМОАССОЦИАЦИИ
}

\author{
(Представил О. Эйзен)
}

Как известно, физико-химический анализ в классической трактовке заключается в исследовании зависимости какого-нибудь свойства системы от ее состава. На основе анализа форм изотермы свойства $Y$ или отклонения их от аддитивности $\Delta Y$ можно получить информацию о процессах, протекающих в системе. Если в этой системе имеет мести лишь какая-нибудь одна реакция, то для мольного свойства экстремум функции $\Delta Y$ в диаграмме «состав-свойство» соответствует стехиометрическому соотношению взаимодействующих компонентов, что дает возможность установить состав образующихся соединений и рассчитать соответствующие константы равновесия [1-4]. При этом активности компонентов системы обычно считают пропорциональными их концентрациям, т. е. концентрационной зависимостью коэффициентов активности пренебрегают.

В настоящей работе показывается, что в системе, где один компонент склонен к самоассоциации (а тем более, оба), такая трактовка диаграмм «состав-свойство» может привести к ошибочным выводам. причем иногда можно «обнаружить» образование таких соединений, существование которых не поддается доказательству другими методами и противоречит общим представлениям химии.

В частности, к таким системам относятся смеси, содержащие карбоновые кислоты, фенолы, спирты, амины и другие соединсния, склонные к образованию водородной связн.

Рассмотрим систему, состоящую из неассоциированного компонента $A$ и ассоциированного $B$. Для простоты математической формулировки задачи примем, что вклад любой формы молекулярного агрегата в величину измеряемого свойства $Y$ пропорционален концентрации этого агрегата в системе. Допустим также, что коэффициенты активности любого агрегата не зависят от состава системы и что $A$ и $B$ не взаимодействуют друг с другом. Тогда свойство $Y$ при мольных долях компонентов, равных $x_{A}$ и $x_{B}$, выражается

или

$$
Y=Y_{A} x_{A}+Y_{B_{1}} x_{B_{1}}+Y_{B_{2}} x_{B_{2}}+\ldots+Y_{B_{L}} x_{B_{L}}+\ldots
$$

$$
Y=Y_{A} x_{A}+\sum_{L=1}^{p} Y_{B_{L}} x_{B_{L}}
$$

где $x_{B_{1}}, x_{B_{2}}, \ldots, x_{B_{L}}-$ мольная доля агрегатов $B_{1}, B_{2}, \ldots, B_{L}$, отнесенная к одному стехиометричсскому молю системы; $Y_{B_{1}}, Y_{B_{2}}, \ldots, Y_{B_{L}}-$ свойство $Y$, проявляемое агрегатами $B_{1}, B_{2}, \ldots, B_{L} ; p-$ количество мономеров $B$ в агрегате $B_{L}$ с наивысшим $L$. 
Выразив $x_{B_{L}}$ через соответствующие константы самоассоциации $\left(K_{11}=1, \quad K_{12}=x_{B_{2}} / x_{B_{1}}^{2}, \ldots, K_{1 L}=x_{B_{L}} / x_{B_{1}}^{L}\right), \quad$ запишем уравнение (1) в виде

$$
Y=Y_{A} x_{A}+\sum_{L=1}^{p} Y_{B_{L}} K_{1 L} x_{B_{1}}^{L}
$$

Нетрудно убедиться, что строгая аддитивность свойства $Y$ составу $x_{B}$ сохраняется только в том случае, если во всем интервале концентраций $\left(0 \leqslant x_{B} \leqslant 1\right) \quad Y_{B_{L}}=$ const,

$$
\frac{\mathrm{d} Z}{\mathrm{~d} x_{B}} \equiv \frac{\mathrm{d}}{\mathrm{d} x}\left(\sum_{L=1}^{p} Y_{B_{L}} K_{1 L} x_{B_{1}}^{L}\right)=\text { const и } \frac{\mathrm{d}^{2} Z}{\mathrm{~d} x_{B}^{2}}=0 .
$$

Если при любом значении $x_{B}$ вторая производная функции $Z$ отрицательна, имеет место положительное отклонение $Y$ от аддитивности. Отрицательное отклонение характерно для систем, для которых $\frac{\mathrm{d}^{2} Z}{\mathrm{~d} x_{B}^{2}}>0$. S-образная форма кривой $Y$ наблюдается в таких системах, где $\frac{\mathrm{d}^{2} Z}{\mathrm{~d} x_{B}^{2}}$ изменяет знак.

Ниже будет показано, что при всех наиболее вероятных схемах самоассоциации компонента $B \frac{\mathrm{d}^{2} Z}{\mathrm{~d} x_{B}^{2}}<0$, т. е. в системах с одним самоассоциированным компонентом следует ожидать положительного отклонения $Y$ от аддитивности.

Так как зависимость $Z$ (или $Y$ ) от $x_{B}$ обычно очень трудно выразить в явной форме, математическое решение проблемы заключается в дифференцировании неявной функции $F\left(x_{B}, Z\right)=0$.

\section{1. Образование только одного самоассоциата $B_{L}$}

1.1. $\boldsymbol{B}_{L}$ не обладает свойством $\boldsymbol{Y}\left(\boldsymbol{Y}_{B_{L}}=0\right)$. Такой случай реализуется, если образуется циклический ассоциат, причем свойство $Y$ отражает налуячие свободных концевых групп. Тогда

$$
Z=Y_{B_{1}} x_{B_{1}} \text {. }
$$

С другой стороны, из материального баланса имеем

или

$$
x_{B}=x_{B_{1}}+L K_{1 L} x_{B_{1}}^{L}
$$

$$
x_{B}-x_{B_{1}}\left(1+L K_{1 L} x_{B_{1}}^{L-1}\right)=0 .
$$

При дифференцировании этой неявной функции $F\left(x_{B}, x_{B_{1}}\right)=0$ по $x_{B}$ и $x_{B_{1}}$ получим

$$
\begin{gathered}
F_{x_{B}}^{\prime}=1, \quad F_{x_{B_{t}}}^{\prime}=-\left(1+L^{2} K_{1 L} x_{B_{1}}^{L-1}\right), \quad F_{x_{B} x_{B}}^{\prime \prime}=0, \quad F_{x_{B} x_{B_{1}}}^{\prime \prime}=0, \\
F_{x_{B_{1}} x_{B_{1}}}^{\prime \prime}=-L^{2}(L-1) K_{1 L} x_{B_{1}}^{L-2}
\end{gathered}
$$

откуда легко можно найти

$$
\frac{\mathrm{d}^{2} Z}{\mathrm{~d} x_{B}^{2}}=Y_{B_{1}} \frac{\mathrm{d}^{2} x_{B_{1}}}{\mathrm{~d} x_{B}^{2}}=-Y_{B_{1}} \frac{L^{2}(L-1) K_{1 L} x_{B_{1}}^{L-2}}{\left(1+L^{2} K_{1 L} x_{B_{1}}^{L-1}\right)^{3}},
$$

из которого вытекает $\frac{\mathrm{d}^{2} Z}{\mathrm{~d} x_{B}^{2}}<0$. 
1.2. $\boldsymbol{B}_{L}$ обладает свойством $\boldsymbol{Y}$ аналогично мономеру $\boldsymbol{B}_{1}\left(\boldsymbol{Y}_{B_{L}}=\boldsymbol{Y}_{B_{1}}>0\right)$. Такой случай реализуется, если свойство $Y$ овязано с наличием свободных концевых групп как в мономере, так и в образующемся (линейном) ассоциате. Тогда

$$
Z=Y_{B_{1}} x_{B_{1}}+Y_{B_{L}} K_{1 L} x_{B_{1}}^{L}=Y_{B_{1}}\left(x_{B_{1}}+x_{B_{L}}\right)
$$

или

$$
Z=Y_{B_{1}} N
$$

где $N \equiv \sum_{L=1}^{p} x_{B_{L}}-$ фактическая концентрация агрегатов компонента $B$, отнесенная к одному стехиометрическому молю системы.

Учитывая, что $x_{B}=\sum_{L=1}^{p} L x_{B_{L}}$, легко получаем

$$
K_{1 L}=\frac{(L-1)^{L-1}\left(x_{B}-N\right)}{\left(L N-x_{B}\right)^{L}},
$$

откуда после несложного преобразования находим функцию $F\left(x_{B}, N\right)$, связывающую $x_{B}$ и $N$ в неявном виде

$$
F\left(x_{B}, N\right)=K_{1 L}\left(L N-x_{B}\right)^{L}-(L-1)^{L-1} x_{B}+(L-1)^{L-1} N=0 .
$$

При дифференцировании $F\left(x_{B}, N\right)$ по $x_{B}$ и $N$ получим

$$
\begin{aligned}
& F_{x_{B}}^{\prime}=-\left[K_{1 L} L\left(L N-x_{B}\right)^{L-1}+(L-1)^{L-1}\right], \\
& F_{N}^{\prime}=K_{1 L} L^{2}\left(L N-x_{B}\right)^{L-1}+(L-1)^{L-1} \\
& F_{x_{B} x_{B}}^{\prime \prime}=K_{1 L} L(L-1)\left(L N-x_{B}\right)^{L-2} \\
& F_{N N}^{\prime \prime}=K_{1 L} L^{3}(L-1)\left(L N-x_{B}\right)^{L-2} \\
& F_{x_{B} N}^{\prime \prime}=-K_{1 L} L^{2}(L-1)\left(L N-x_{B}\right)^{L-2}
\end{aligned}
$$

и

$$
\frac{\mathrm{d}^{2} Z}{\mathrm{~d} x_{B}^{2}}=Y_{B_{1}} \frac{\mathrm{d}^{2} N}{\mathrm{~d} x_{B}^{2}}=-\mathrm{Y}_{B_{1}} K_{1 L} L(L-1)^{2 L+1}\left(L N-x_{B}\right)^{L-2}\left(F_{N}^{\prime}\right)^{-3}
$$

Учитывая, что $L N-x_{B}>0$, нетрудно убедиться, что $F_{N}^{\prime}>0$ и $\frac{\mathrm{d}^{2} Z}{\mathrm{~d} x_{B}^{2}}<0$

Аналогичным образом можно показать, что такая же закономерность сохраняется для систем, в которых полимеризация компонента $B$ ведет к образованию нескольких ассоциатов (напр., ди- и тетрамеров).

\section{2. Цепочечная самоассоциация при постоянстве константы равновесия для реакции присоединения мономера (схема Мекке-Кемптера)}

2.1. Самоассоциаты не обладают свойством $Y$. В этом случае единственным агрегатом $B_{L}$, обладающим свойством $Y$, является мономер $B_{1}$ и

$$
Z=Y_{B_{1}} x_{B_{1}}
$$

С другой стороны, если $K_{12}=K_{23}=\ldots=K_{(L-1) L}=\ldots$ и $p=\infty$, то 


$$
x_{B}=\sum_{L=1}^{\infty} L K_{12}^{L-1} x_{B_{1}}^{L}=\frac{x_{B_{1}}}{\left(1-K_{12} x_{B_{1}}\right)^{2}},
$$

откуда получим функцию

$$
F\left(x, x_{B_{1}}\right)=x_{B}-\left(2 K_{12} x_{B}+1\right) x_{B_{1}}+K_{12}^{2} x_{B} x_{B_{1}}^{2}=0 .
$$

При дифференцировании уравнения (11) по $x_{B}$ и $x_{B_{1}}$ находим

$$
\begin{aligned}
& F_{x_{B}}^{\prime}=\left(1-K_{12} x_{B_{1}}\right)^{2}, \\
& F_{x_{B_{1}}}^{\prime}=2 K_{12}\left(K_{12} x_{B_{1}}-1\right) x_{B}-1, \\
& F_{x_{B} x_{B}}^{\prime \prime}=0 \\
& F_{x_{B_{1} x_{B_{1}}}}^{\prime \prime}=2 K_{12}^{2} x_{B} \\
& F_{x_{B} x_{B_{1}}}^{\prime \prime}=2 K_{12}\left(K_{12} x_{B_{1}}-1\right)
\end{aligned}
$$

и

$$
\begin{gathered}
\frac{\mathrm{d}^{2} Z}{\mathrm{~d} x_{B}^{2}}=Y_{B_{\mathrm{t}}} \frac{\mathrm{d}^{2} x_{B_{1}}}{\mathrm{~d} x_{B}^{2}}= \\
=-2 Y_{B_{1}} K_{12}\left(1-K_{12} x_{B_{1}}\right)^{3} \frac{3 K_{12}\left(K_{12} x_{B_{1}}-1\right) x_{B}-2}{\left[2 K_{12}\left(K_{12} x_{B_{1}}-1\right) x_{B}-1\right]^{3}} .
\end{gathered}
$$

Так как $K_{12} x_{B_{1}}-1<0$, нетрудно убедиться, что $\frac{\mathrm{d}^{2} Z}{\mathrm{~d} x_{B}^{2}}<0$.

2.2. Все самоассоциаты обладают свойством $\boldsymbol{Y}$. Предполагая $Y_{B_{1}}=$ $=Y_{B_{2}}=\ldots=Y_{B_{L}}=\ldots$, можем написать

$$
Z=Y_{B_{1}} \sum_{L=1}^{\infty} x_{B_{L}}=Y_{B_{1}} N
$$

Поскольку

$$
N=\sum_{L=1}^{\infty} K_{12}^{L-1} x_{B_{1}}^{L}=\frac{x_{B_{1}}}{1-K_{: 2} x_{B_{1}}}, .
$$

то путем комбинирования уравнений (10) и (14) находим

откуда получим

$$
K_{12}=\left(x_{B}-N\right) / N^{2},
$$

$$
F\left(x_{B}, N\right)=N\left(K_{12} N+1\right)-x_{B}=0 .
$$

При дифференцировании этой неявной функции по $x_{B}$ и $N$ легко найти, что

и

$$
\frac{\mathrm{d}^{2} Z}{\mathrm{~d} x_{B}^{2}}=Y_{B_{1}} \frac{\mathrm{d}^{2} N}{\mathrm{~d} x_{B}^{2}}=-Y_{B_{1}} \frac{-2 K_{12}}{\left(2 K_{12} N+1\right)^{3}}
$$

$$
\frac{\mathrm{d}^{2} Z}{\mathrm{~d} x_{B}^{2}}<0 .
$$

\section{3. Цепочечная самоассоциация при непостоянстве константы равновесия для реакции присоединения мономера (схема Лассетра)}

Допустим, что все самоассоциаты обладают свойством $Y$, причем $Y_{B_{1}}=Y_{B_{2}}=\ldots=Y_{B_{L}}=\ldots$. Тогда аналогично случаю 2.2 находим

$$
Z=Y_{B_{1}} \sum_{L=1}^{\infty} x_{B_{L}}=Y_{B_{1}} N
$$




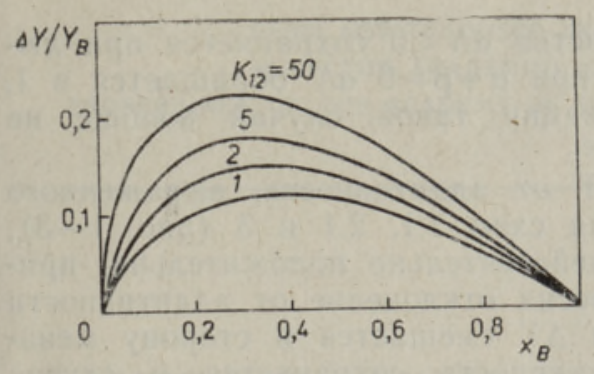

Рис. 1. Зависимость $\Delta Y / Y_{B}$ от $x_{B}$ при димеризации компонента $B\left(Y_{B}-\right.$ величина свойства $\hat{Y}$ при $\left.x_{B}=1\right)$.

Рис. 2. Зависимость $\Delta Y / Y_{B}$ от $x_{B}$ при цепочечной самоассоциации компонента $B$ по схеме Мекке-Кемптера.

Рис. 3. Зависимость $\Delta Y / Y_{B}$ от $x_{B}$ при цепочечной самоассоциации по схеме Лассетра.
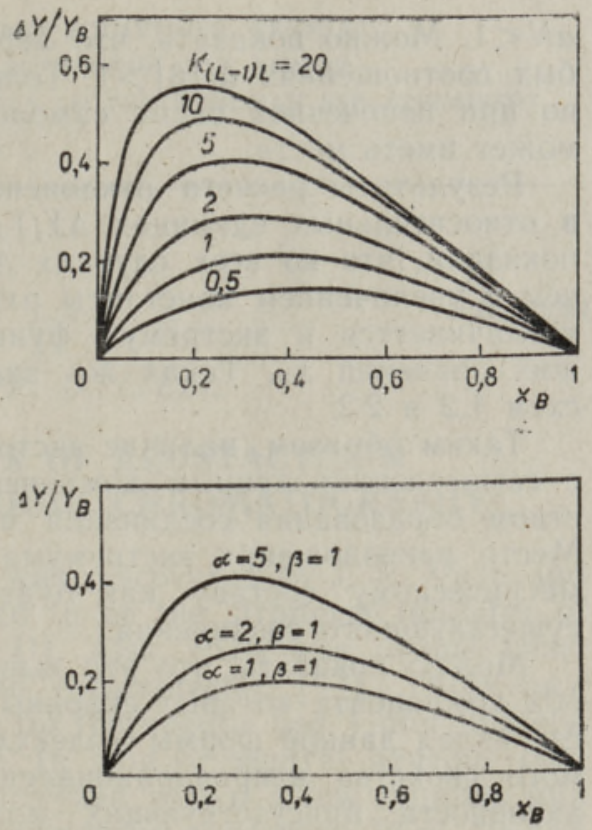

Используя согласно [5] для описания зависимости между $x_{B}$ и $N$ линейное уравнение с двумя эмпирическими константами $(\alpha$ и. $\beta)$ вида

находим

$$
x_{B} / N=1+\alpha x_{B}+\beta N \text {, }
$$

и

$$
\frac{\mathrm{d} x_{B}}{\mathrm{~d} N}=\frac{1+\beta N}{1-\alpha N}+N \frac{\alpha+\beta}{(1-\alpha N)^{2}}
$$

$$
\frac{\mathrm{d}^{2} x_{B}^{\prime}}{\mathrm{d} N^{2}}=\frac{2(\alpha+\beta)}{(1-\alpha N)^{3}}
$$

Нетрудно убедиться, что $\frac{\mathrm{d}^{2} Z}{\mathrm{~d} x_{B}^{2}}=Y_{B_{1}} \frac{\mathrm{d}^{2} N}{\mathrm{~d} x_{B}^{2}}<0, \quad$ если $\frac{\mathrm{d}^{2} x_{B}}{\mathrm{~d} N^{2}}>0$. Поскольку $\alpha+\beta \equiv K_{12}>0$, то это. отвечает действительности, если $1-\alpha N>0$.

При $\alpha \leqslant 0$ правильность последнего условия очевидна. Если же $\alpha>0$, то возможны следующие два случая:

a) $\alpha>0, \beta \geqslant 0$. При этих условиях из уравнения (18) легко найти

$$
1-\alpha N=N x_{B}^{-1}(1+\beta N)>0 .
$$

б) $\alpha>0, \beta<0$. Решая уравнение (18) относительно $N$, получим

$$
\alpha N=\frac{\alpha}{2 \beta}\left[\left(1+\alpha x_{B}\right)-\sqrt{\left(1+\alpha x_{B}\right)^{2}+4 \beta x_{B}}\right],
$$

анализ которого показывает, что неравенство $\alpha N<1$ справедливо при любых значениях $\alpha$ и $\beta$, если толыко $\alpha+\beta>0$.

Действительно, если $\alpha \gg|\beta|$, то получим *

$$
\alpha N \approx \frac{\alpha x_{B}}{1+\alpha x_{B}}<1 \text {. }
$$

Если же $\alpha$ и $|\beta|$ отличаются несущественно (т. е. величина $K_{12} \equiv \alpha+\beta$ очень мала), то $-\alpha / 2 \beta \approx 1 / 2$, но $\left(1+\alpha x_{B}\right)-\sqrt{\left(1+\alpha x_{B}\right)^{2}+4 \beta x_{B}}<2$ и

* В общем случае, если $a \gg b$, то $\sqrt{a^{2}+b \approx} a+b / 2 a$. 
$\alpha N<1$. Можно показать, что неравенство $\alpha N<0$ сохраняется при любых соотношениях $\alpha /|\beta|>1$. Только при $\alpha+\beta=0 \alpha N$ обращается в 1 , но при цепочечной схеме самоассоциации такой случай вообе не может иметь места.

Результаты расчета отклонения $Y$ от аддитивности, выраженного в относительных единицах $\Delta Y / Y_{B}$ для схем 1.1, 2.1 и 3 (рис. 1-3), показали, что во всех случаях $\Delta Y$ действительно положительна, причем с увеличением константы равновесия отклонение от аддитивности увеличивается и экстремум функции $\Delta Y$ смещается в сторону меньших значений $x_{B}$. Такая же закономерность сохраняется в случае схем 1.2 и 2.2 .

Таким образом, наличие экстремума $Y$ или $\Delta Y$ в двойных системах с самоассоциированным компонентом не может служить доказательством образования соединения между компонентами системы $A$ и $B$. Место расположения экстремума не должно соответствовать стехиометрическому составу как будто образовавшегося, но реально не существующего соединения.

Можно показать, что эти выводы распространяются и на системы, оба компонента которых склонны к самоассоциации. В случае систем, где вклад данной формы молекулярного агрегата в величину измеряемого свойства непропорционален концентрации и/или коэффициенты активности присутствующих молекулярных агрегатов зависимы от состава системы, правомерность изложенных положений также очевидна.

Л И Т Р А Т У Р А

1. Усанович М. И. О диаграммах физико-химического анализа двойных жидких систем. - Докл. АН СССР, 1935, 1, № 1, 378-381.

2. Фиалков Ю. Я. Двойные жидкие системы. Киев, 1969.

3. Фиалков Ю. Я., Фенерли Г. Н. Метрика диаграмм двойных жидких систем с аддационным взанмодействием. - Ж. неорган. хим., 1966, 11, № 7, 1708-1710.

4. Аносов В. Я., Озерова М. Н., Фиалков Ю. Я. Оеновы физико-химического анализа. М., 1976.

5. Lassetre, E. N. Interpretation of freezing-point lowering data in terms of polymerization. - J. Amer. Chem. Soc., 1937, 59, N 7, 1383-1387.

Ннститут химии

Академии наук Эстонской ССР
Поступила в редакцию 4/I 1984

\section{MOLDER, H. TAMVELIUS}

\section{OHE AUTOASSOTSIEERUVA KOMPONENDIGA BINAARSETE VEDELSUSTEEMIDE FUOSIKALIS-KEEMILISE ANALOUSI PROBLEEMID}

On tõestatud, et autoassotsieeruvat komponenti sisaldavate binaarsete vedelsüsteemide omaduse-koostise diagrammil peab esinema maksimum. Ekstreempunkti olemasolu diagrammil ei ole aga piisav tõendamaks, et segu komponentidest moodustub kindla koostisega ühend.

\section{MOLDER, H. TAMVELIUS}

\section{THE PHYSICO-CHEMICAL ANALYSIS OF BINARY LIQUID SYSTEMS CONTAINING A SELF-ASSOCIATING COMPONENT}

An inevitable appearance of a maximum on the quality - composition diagram of binary liquid systems containing a seli-associating component is demonstrated. The existence of such a maximum reflects the self-association but is not sufficient to verify the formation of a compound with fixed composition. 